\title{
HTLV-I associated Sjögren's syndrome is aetiologically distinct from anti-centromere antibodies positive Sjögren's syndrome
}

\author{
Ayumi Hida, Yojiro Kawabe, Atsushi Kawakami, Kiyoshi Migita, Masahiro Tominaga, \\ Hideki Nakamura, Katsumi Eguchi
}

\begin{abstract}
Objective-To investigate whether Sjögren's syndrome (SS) with anti-HTLV-I antibodies is aetiopathologically distinguishable from SS without these antibodies, the study compared prevalence of autoantibodies in serum samples of SS patients with or without anti-HTLV-I antibodies.

Methods-The test group included 135 patients with primary SS and 97 patients with secondary SS. Serum samples of the patients were examined for the presence of anti-nuclear antibodies (ANA), anti-SSA/Ro antibodies, anti-SS-B/La antibodies, anti-centromere antibodies (ACA), and anti-HTLV-I antibodies.
\end{abstract}

Results-Anti-HTLV-I antibodies were detected in $25.0 \%$ of primary SS patients and in $29.2 \%$ of secondary SS patients. There were no significant differences in the mean age, sex, values of asparate aminotransferase, alanine aminotransferase, alkaline phosphatase, serum complements and IgG between HTLV-I seropositive and seronegative SS patients. The rheumatoid factor, ANA, anti-SS-A/Ro, and anti-SS-B/La antibodies in serum samples of SS patients were detected in $60.0 \%, 84.0 \%, 51.9 \%$, and $12.0 \%$, respectively. There was no significant difference in the prevalence of these antibodies between HTLV-I seropositive and seronegative SS patients. Using the indirect immunofluorescence test, $14.2 \%$ showed a discrete speckled staining pattern. All serum samples contained significant amounts of ACA determined by enzyme linked immunosorbent assay. These antibodies were detected in only $4 \%$ of HTLV-I seropositive SS patients but were present in $19.9 \%$ of HTLV-I seronegative SS patients. Furthermore, the prevalences of anti-SS-A/Ro and anti-SS-B/La antibodies in serum samples of ACA positive patients were significantly lower than those in ACA negative SS patients.

Conclusion-These results suggest that SS patients with anti-SS-A/Ro or anti-SSB/La antibodies, or both, might be aetio- pathologically distinct from SS patients with ACA. HTLV-I might be involved in the pathogenesis of $\mathrm{SS}$ in a subset of patients with anti-SS-A/Ro or anti-SSB/La antibodies, or both, but not SS patients with ACA.

(Ann Rheum Dis 1999;58:320-322)

Sjögren's syndrome (SS) is an autoimmune disorder characterised by chronic lymphocytic infiltration of salivary and lacrimal glands, and systemic production of autoantibodies. ${ }^{1}$ The exact pathogenic mechanisms of SS remain to be established, but viral infections have been considered as a possible aetiological factor. In this regard, previous studies have suggested a correlation between SS and human T lymphotropic virus type I (HTLV-I). ${ }^{2-4}$ We have reported a high seroprevalence of HTLV-I in female SS patients, compared with female blood donors, residing in Nagasaki, a city located in the south western part of Japan and is heavily endemic for HTLV-I infection. ${ }^{4}$ Furthermore, a proportion of HTLV-I infected patients with tropical spastic paraparesis (TSP) ${ }^{2}$ and HTLV-I associated myelopathy (HAM) ${ }^{5}$ also develop clinical features of SS. Salivary IgA class antibodies to HTLV-I are also commonly detected in HTLV-I seropositive patients with SS. ${ }^{4}$ These findings suggest a close relation between HTLV-I infection and SS in endemic areas, and that HTLV-I might induce SS. Characterisation of the differences in serological and laboratory findings between HTLV-I seropositive and HTLV-I seronegative patients with SS is important in the investigation of the pathophysiological mechanisms of SS. We have previously reported a high incidence of extraglandular manifestations, such as uveitis, myelopathy, and recurrent episodes of pyrexia in HTLV-I seropositive SS patients compared with patients who did not have antibodies to HTLV-I. ${ }^{3}$ This study is an extension to our early work and aimed to compare the presence of several autoantibodies in serum samples of HTLV-I seropositive and seronegative SS patients.

\section{Methods}

The test group included 135 patients with primary SS (8 men and 127 women; 58.7 (13.0) 
Table 1 Clinical features of patients with Sjögren's syndrome with or without anti-HTLV-I Abs

\begin{tabular}{lll}
\hline & $\begin{array}{l}\text { Patients with } \\
\text { Abs to HTLV-I }\end{array}$ & $\begin{array}{l}\text { Patients without } \\
\text { Abs to HTLV-I }\end{array}$ \\
\hline Patients (n) & 50 & 136 \\
Age (y) & $60.3(12.4)$ & $56.9(12.8)$ \\
Sex (male: female) & $4: 46$ & $10: 126$ \\
AST (IU/1) & $26.1(21.1)$ & $26.5(22.7)$ \\
ALT (IU/1) & $17.9(21.1)$ & $23.2(36.5)$ \\
Alp (IU/1) & $196.4(82.8)$ & $219.4(214.5)$ \\
IgG (mg/d) & 2409.7 & 2394.4 \\
Serum complement & $(949.1)$ & $(1069.1)$ \\
$\quad$ concentration (CH50/ml) & $37.0(5.1)$ & $36.4(7.7)$ \\
\hline
\end{tabular}

Data shown as mean (SD).

years (mean (SD)) and 97 patients with secondary SS (10 men and 87 women, age; 56.6 (13.0) years). All patients fulfilled the preliminary criteria for the diagnosis of SS as set out by the European Community. ${ }^{6}$ Patients with secondary SS consisted of 43 patients with rheumatoid arthritis (RA), 25 patients with systemic lupus erythematosus (SLE), 17 patients with mixed connective tissue disease (MCTD), 10 patients with progressive systemic sclerosis (PSS), two patients with polymyositis (PM), and one patient with polyarteritis nodosa $(\mathrm{PN})$.

Informed consent was obtained from all the subjects examined, and the study was conducted in accordance with human experimentation guidelines of Nagasaki University School of Medicine.

Anti-nucleur antibodies (ANA) were detected with an indirect immunofluorescence procedure using Hep-2 cells (Fluoro Hep Ana test, Medical and Biological Laboratories; MBL, Nagoya, Japan). Antibodies to SS-A/Ro, SS-B/La and centromere antigens were determined by enzyme linked immunosorbent assay (ELISA) (Mesacup SS-A/Ro test, Mesacup SS-B/La test, Mesacup CENP-B test, MBL). Antibodies to HTLV-I were measured by the particle agglutination assay (Serodia-ATL kit, Fuji Rebio, Japan) and ELISA (Eitest-ATL test, Eisai, Tokyo, Japan). Serum samples positive for anti-HTLV-I antibodies were further confirmed by western blot analysis using a commercial immunoblotting kit (Problot, HTLV-I, Fuji Rebio, Japan).

Differences between groups were analysed for statistical significance using Student's $t$ test, $\chi^{2}$ test or Fisher's exact test. A p value less than 0.05 denoted the presence of a statistically significant difference.

\section{Results}

Anti-HTLV-I antibodies were detected in 50 of 186 serum samples $(26.9 \%)$ of patients with both primary and secondary SS. Table 1 shows the clinical features of patients with SS with or without anti-HTLV-I antibodies. There were no significant differences in the mean age, sex, values of aspartate aminotransferase, alanine
Table 3 Prevalence of $A b$ s to SS-A/Ro and SS-B/La in patients with $S S$ with $A C A$ and those without $A C A$

\begin{tabular}{lll}
\hline ACA & Abs to SS- A/Ro & Abs to SS-B/La \\
\hline Seropositive group & $6 / 28(21.4 \%)$ & $0 / 28(0 \%)$ \\
Seronegative group & $98 / 174(56.3 \%)$ & $23 / 174(13.2 \%)$ \\
Statistical analysis & $\mathrm{p}=0.0013$ & $\mathrm{p}=0.0495$ \\
\hline
\end{tabular}

aminotransferase, alkaline phosphatase, serum complements and serum IgG concentration between HTLV-I seropositive and seronegative SS patients.

Rheumatoid factor and ANA were detected in serum samples of 99 of 165 patients $(60.0 \%)$ and 194 of 232 patients (84.0\%), respectively. A speckled pattern was observed in 117 serum samples $(54.0 \%)$, diffuse pattern in 37 serum samples $(15.9 \%)$, and a discrete speckled pattern was detected in 33 serum samples $(14.2 \%)$. Using the ELISA test, anticentromere antibodies (ACA) were detected in all serum samples that exhibited a discrete speckled pattern shown by the indirect immunofluorescence test. Antibodies to SS-A/Ro and SS-B/La antigens were detected in 108 of 208 serum samples $(51.9 \%)$ and in 25 of 208 serum samples $(12.0 \%)$ of SS patients.

Anti-HTLV-I antibodies were detected in 29 of 116 serum samples $(25.0 \%)$ of patients with primary SS, and in 21 of 72 serum samples $(29.2 \%)$ of patients with secondary SS. The proportion of primary SS patients with antiHTLV-I antibodies was not different to those with secondary SS. Among patients with secondary SS, 7 of 31 serum samples $(22.6 \%)$ of RA patients, 6 of 21 serum samples $(28.6 \%)$ of SLE patients, 3 of $12(25.0 \%)$ of MCTD patients, 3 of 7 serum samples (42.9\%) of PSS patients were seropositive for HTLV-I.

Anti-SS-A/Ro antibodies were detected in 19 of 45 serum samples (42.2\%) of HTLV-I seropositive SS patients, and 74 of 124 serum samples $(59.7 \%)$ of HTLV-I seronegative SS patients (table 2). The prevalence of anti-SS-A/Ro antibodies in HTLV-I seropositive SS patients was lower than that in HTLV-I seronegative SS patients, but the difference was not significant. The proportion of HTLV-I seropositive patients with anti-SS-B/La antibodies (5 of 45 serum samples, $11.1 \%$ ) was not different to that in seronegative patients (18 of 124 serum samples, $14.5 \%)$. Furthermore, the proportions of HTLV-I seropositive patients with ANA and rheumatoid factor ( 41 of $50,82.0 \%$ and 20 of 35 serum samples, $57.1 \%$, respectively) were not different to those in seronegative patients (153 of $182,84.1 \%$ and 61 of 103 serum samples, $59.2 \%$, respectively). In contrast, ACA were found in only 2 of 50 serum samples $(4.0 \%)$ of HTLV-I seropositive patients although these were detected in 27 of 136 serum samples (19.9\%) of HTLV-I seronegative patients. The proportion of HTLV-I seropositive SS patients

Table 2 Prevalence of antibodies in serum samples from SS patients with or without anti-HTLV-I Abs

\begin{tabular}{llllll}
\hline Anti-HTLV-I Abs & RF & ANA & Anti-SS-A/Ro Abs & Anti-SS-B/La Abs & ACA \\
\hline Seropositive group & $20 / 35(57.1 \%)$ & $41 / 50(82.0 \%)$ & $19 / 45(42.2 \%)$ & $5 / 45(11.1 \%)$ & $2 / 50(4.0 \%)$ \\
Seronegative group & $61 / 103(59.2 \%)$ & $153 / 182(84.1 \%)$ & $74 / 124(59.7 \%)$ & $18 / 124(14.5 \%)$ & $27 / 136(19.9 \%)$ \\
$\chi^{2}$ test & $\mathrm{p}=0.9862$ & $\mathrm{p}=0.8935$ & $\mathrm{p}=0.0654$ & $\mathrm{p}=0.7502$ & $\mathrm{p}=0.0158$ \\
\hline
\end{tabular}


with ACA was markedly less than that of HTLV-I seronegative SS patients (table 2, $\mathrm{p}=0.0158)$.

Finally, we determined the prevalence of antibodies to SS-A/Ro and SS-B/La after dividing SS patients with or without ACA. Anti-SS-A/Ro antibodies were found in 6 of 28 serum samples $(21.4 \%)$ of ACA positive SS patients but in 98 of 174 serum samples $(56.3 \%)$ of ACA negative SS patients $(\mathrm{p}=0.0013)$. Twenty three of 174 serum samples $(13.2 \%)$ of ACA negative SS patients had anti-SS-B/La antibodies. In contrast, antiSS-B/La antibody was not found in all 28 serum samples of ACA positive SS patients $(p=0.0495)$. The proportions of ACA positive SS patients with antibodies to SS-A/Ro and SS-B/La were statistically different than in those of ACA negative SS patients (table 3).

\section{Discussion}

In this study, we first demonstrated a high prevalence of anti-HTLV-I antibodies in serum samples of patients with primary and secondary SS. Our previous data indicated that the risk of developing SS among HTLV-I seropositive subjects was 3.1 times higher than that among HTLV-I seronegative subjects in Nagasaki City. ${ }^{4}$ No significant differences in the incidence of anti-HTLV-I antibodies were found between primary and secondary SS groups in this study.

We compared the prevalence of autoantibodies in serum samples of HTLV-I seropositive and negative SS patients. The present results confirmed those of a previous study, which included 40 cases of SS, ${ }^{5}$ that the proportions of HTLV-I seropositive patients with rheumatoid factor, ANA, anti-SS-A/Ro antibodies, and anti-SS$\mathrm{B} / \mathrm{La}$ antibodies were not significantly different from those in HTLV-I seronegative SS patients. These results suggest that the above antibodies are commonly produced in serum samples of HTLV-I seropositive SS patients as well as HTLV-I seronegative SS patients. Discrete speckled staining was found in $14.2 \%$ of SS patients. The presence of ACA was verified in all 33 serum samples with discrete speckled staining. The prevalence of ACA in serum samples of SS patients in this study is in agreement with those described previously. ${ }^{7}$ It has been reported $^{8}$ that SS patients with ACA were characterised by a lower incidence of rheumatoid factor, anti-SS-A/Ro, and anti-SS-B/La antibodies than those described in the medical litera- ture. This study demonstrated that the prevalence of ACA in serum samples of HTLV-I seropositive patients is significantly less than in HTLV-I seronegative patients. Considered together, the above findings suggest that the production of ACA is not likely to be implicated in SS associated with HTLV-I infection, and that SS with ACA could be differentiated from HTLV-I positive SS.

We also demonstrated that the prevalence of anti-SS-A/Ro and anti-SS-B/La antibodies in serum samples of ACA positive SS patients was significantly less than that in the antibody negative SS patients. These results strongly supported the hypothesis that SS patients with ACA were aetiopathologically distinct from SS patients without these antibodies, and that HTLV-I associated SS patients were related to SS patients in a subset of patients with anti-SS-A/Ro or anti-SS-B/La antibodies, or both.

In summary, our data strongly indicate that HTLV-I is involved in the pathogenesis of the disease in a subset of patients with SS in endemic areas. The pathological processes may be different between SS patients with anti-SSA/Ro or anti-SS-B/La antibodies, or both, and those with ACA. It seems probable that HTLV-I infection is a causative agent in SS with anti-SS-A/Ro or anti-SS-B/La antibodies, or both, but not in SS with ACA. Elucidation of the relation between HTLV-I infection and SS will provide a new insight to our understanding of the pathogenesis of SS.

1 Talal N, Moutsopoulos HM, Kassan S. Sjögren's syndrome: clinical and immunologic aspects. Heidelberg: Springer Veslag, 1987

2 Vernant JC, Buisson G, Magdeleine J, DeThore J, Jouannelle A, Neisson-Vernant C, et al. T-lymphocyte alveolitis, tropical spastic paresis, and Sjögren's syndrome. Lancet 1988;i:177.

3 Eguchi K, Matsuoka N, Ida H, Nakashima M, Sakai M, Sakito S, et al. Primary Sjögren's syndrome with antibodies to HTLV-I: clinical and to HTLV-I: clinical and

4 Terada K, Katamine S, Eguchi K, Moriuchi R, Kita M, Shimada $\mathrm{H}$, et al. Prevalence of serum and salivary antibodies to HTLV-I in Sjögren's syndrome. Lancet 1994;344:1116 19.

5 Nakamura H, Eguchi K, Nakamura T, Mizokami A, Shirabe S, Kawakami A, et al. High prevalence of Sjögren's syndrome in patients with HTLV-I. Ann Rheum Dis 1997; 56:167-72.

6 Vitali C, Bombardieri S, Moutsopoulos HM, Balestrieri G, Bencivelli W, Bernstein RM, et al. Preliminary criteria for the classification of Sjögren's syndrome. Arthritis Rheum the classification

7 Kita M, Eguchi K, Kawabe Y, Tsuboi M, Kawakami A Nakamura $\mathrm{H}$, et al. Abnormal liver function in patients with Nakamura H, et al. Abnormal liver function in patients with
Sjögren's syndrome. Acta Med Nagasaki 1997;41:31-7. Sjögren's syndrome. Acta Med Nagasaki 1997;41:31-7.
Caramaschi P, Biasi D, Carletto A, Manzo T, Randon M, 8 Caramaschi P, Biasi D, Carletto A, Manzo T, Randon M, antibodies. Rev Rhum Engl Ed 1997;64:785-8. 